\title{
Primary care physicians and the management of pediatric concussion: A quandary
}

\author{
Bruce Taubman, MD* \\ Advocare Cherry Hill Pediatric Group, Perelman School of Medicine, Department of General Pediatrics, University of Pennsylvania, USA
}

The need for primary care physician to care for the concussed pediatric patient seems obvious. It has been estimated that there may be as many as 140,000 Emergency Room visits a year for pediatric concussions in the United States [1]. In my primary care pediatric practice only $20 \%$ of concussed patients present initially to the emergency room. If this reflects the experience of the country, there are at least 700,000 episodes of pediatric concussions a year. There are not enough sports medicine physicians or child neurologist to care for every concussed child.

The primary care pediatric physician should be able to care for the concussed patient. The only diagnostic tool needed in the management of concussion is computerized cognitive testing. The most popular one is the ImPact test, which any physician can obtain for use in their office and is readily available in many communities across the United Stated through the schools.

The problem is that none of the many guidelines published by various societies or in review articles are sufficiently data based. It is impossible for the practitioner to practice evidence based medicine when treating concussion as there is no evidence on which to base it.

Although $35 \%$ of concussions are not sports related, all the societies and most of the reviews discuss only sport-concussions. There is no data to show that the mechanism of injury or recovery in sportconcussions differs from the non-sports concussion. In this editorial, we assume that the management should be the same for both.

In 2001 , the $1^{\text {st }}$ international symposium on sport concussion took place in Vienna [2]. A summary published in 2002 recommended rest for the post concussed patient until asymptomatic. No data was presented in the summary to justify this recommendation. In 2010 Grady wrote a review article recommending complete physical and cognitive rest until the patient is asymptomatic [3]. Again, no data is given to justify this. In 2012 the $4^{\text {th }}$ international Conference on Concussion in Sport was held [4]. A consensus statement from the conference stated that the cornerstone of concussion management was physical and cognitive rest. However, it also stated that there is no data on how long the period of rest should be and stressed the need for research in this area.

Between 2012 to the present there has been a dramatic change in the recommendations for pediatric concussion management. In 2013, the American Academy of Pediatrics published a clinical report recommending a protocol to return the concussed patient to the classroom [5]. In this report, cognitive and physical rest until symptom free was no longer recommended. They recommend the exact opposite, patients should return to school while still symptomatic but with accommodations. They gave no data to support this radical change in concussion management. The report has 18 references. All but one are reviews or recommendations by sports medicine specialist. The only research article referenced is by Moser, et al. which documents the efficacy of cognitive and physical rest [6]. In 2013, the American Medical Society for Sports Medicine published a position statement on concussions also recommending patients return to school while symptomatic with accommodations [7]. This recommendation has continued to the present time without any studies comparing the original recommendation, rest until symptom free to the current one, return to school while symptomatic but with accommodations [8]. To add to the practitioner's dilemma the Australian Institute of Sports Medicine and Medical Association and the Australian Medical Association 2017 statement on concussions recommends physical and cognitive rest until symptom free [9].

What are the arguments given for returning concussed patients to school while still symptomatic? The study most often referenced to defend this position is by Thomas, et a.l [10]. In their study 2 days of cognitive and physical rest were compared to 5 days of cognitive and physical rest. Outcome was patient's symptoms at day 10 postconcussion. In both groups a large portion of patients returned to school still symptomatic and were still symptomatic at day 10 . They reported that the group with 5 days of rest had higher symptom scores and slower resolution of symptoms. However, there is no data to suggest that the symptoms score at day 10 post-concussion is predictive of when the patient will become symptom free and functioning at his pre-concussed academic level. Yet this is the goal of concussion management for the primary care practitioner, to have the patient become symptom free and return to his pre-concussion academic level as quickly as possible.

A second argument is that keeping patients home until symptom free results in anxiety and depression. DeFrazio, et al. makes the strongest argument that cognitive and physical rest can be harmful [11]. He references psychological literature which demonstrates that isolating people from normal life activities can cause anxiety and depression and reinforce the idea to patients that they are not well. Unfortunately for the practitioner no studies have been done in the concussed patient. No studies have looked at the psychological effects of having the concussed patient not return to school until symptom free. Nor has any study examined the psychological ramifications of

Correspondence to: Bruce Taubman, MD, Advocare Cherry Hill Pediatric Group, Perelman School of Medicine, Department of General Pediatrics, University of Pennsylvania, USA, E-mail: TAUBMAN@email.chop.edu

Received: June 16, 2017; Accepted: June 28, 2017; Published: June 30, 2017 
having a symptomatic concussed patient attend school but not be able to participate in class work, read, do homework or take tests.

The many guidelines which now recommend returning concussed patients to school with symptoms are making this recommendation based on the clinical experience of the specialist [7]. The problem is that the type of patients seen by a sports medicine clinic are very different than those seen be a primary care physician. The most recent article to give a good description of the demographic of concussed patient at presentation seen in a specialty clinic is Corwin, et al. [12]. The median time between injury and the first visit to their clinic was 14 days, with a range of 1-750 days. In my primary care pediatric office, it is rare for patient to be seen more than 7 days after injury. In their study $46 \%$ of the patients had at least on previous concussion. In my practice $26 \%$ of our patients had a previous concussion [13]. Their patients were referred by another physician whereas in my practice almost all of the concussed patient have been followed by me for routine pediatric care.

Given the difference in 2 patient populations it is not surprising that the clinical experience of the physician in each group may differ. My patients are told that my goal and theirs are the same, to return them to their pre-concussion academic performance as quickly as possible. I believe to do this they need to do cognitive rest for until they are symptom free for up to 3 weeks. If they are still symptomatic after 3 weeks, home tutoring is begun. I see them weekly and support them both medically and emotionally. Using this approach only $17 \%$ of my patients are still symptomatic at 30 days versus $73 \%$ of Corwin's patients [14]. In addition, $63 \%$ reported a decline in grades. This is not surprising given the findings by Ranson, et al. that the more symptoms a post-concussed patient had while in school the more poorly they performed academically [15].

In summary, my own clinical experience of 45 years and my recent studies of 95 patients in our primary care office suggest that post-concussive pediatric patients should not return to the classroom until they are asymptomatic. I believe this approach is more likely to achieve the goal of patients becoming symptom-free and returning to pre-concussion academic levels as quickly as possible. This should also be the outcome for any study evaluating the effectiveness of concussion management. At present there are no conclusive, evidence-based studies demonstrating the superiority of either approach.

I would prefer not to use clinical experience in deciding how to manage the concussed patient, for unexamined clinical experience can lead one to practicing poor medicine [16]. I would much prefer to practice evidence based medicine. Unfortunately, until there is a randomized study comparing rest until symptom free versus return to school with symptoms, in a population similar to the one seen by a primary practitioner, this is not possible.

\section{References}

1. Centers for Disease Control and Prevention (2011) Nonfatal traumatic brain injuries related to sports and recreation activities among persons aged $</-19$ years United Stated, 2001-2009. MMWR Morb Mortao Wkly Rep 60: 1-12

2. Aubry M, Cantu R, Dvorak J, Graf-Baumann T, Johnston KM, et al. (2002) Summary and agreement statement of the 1st International Symposium on Concussion in Sport, Vienna 2001. Clin J Sport Med 12: 6-11. [Crossref]

3. Grady MF (2010) Concussion in the adolescent athlete. Curr Probl Pediatr Adolesc Health Care 40: 154-169. [Crossref]

4. McCrory P, Meeuwisse WH, Aubry M (2013) Consensus statement on concussion in sport: the 4th International conference on concussion in sport held in Zurich. Brt J Sport Med 47: 250-258.

5. Halstead ME, McAvoy K, Devore CD, Carl R, Lee M, et al. (2013) Returning to learning following a concussion. Pediatrics 132: 948-957. [Crossref]

6. Moser RS, Glatts C, Schatz P (2012) Efficacy of immediate and delayed cognitive and physical rest for treatment of sports -related concussion. JPeds 161: 922-926.

7. Harmon KG, Drezner JA, Gammons M (2013) American Medical Society for Sports Medicine position statement: concussion in sport. Br J Sports Med 47: 15-26.

8. McCrea MA, Nelson LD, Guskiewicz K (2017) Diagnosis and Management of Acute Concussion. Phys Med Rehabil Clin N Am 28: 271-286. [Crossref]

9. Elkington JE, Hughes D (2017) Australian Insititute of Sport and Australian Medical Association position statement on concussion in sport-medicine. MJA 206: 46-50.

10. Thomas DG, Apps JN, Hoffman RG, McCrea M, Hammeke T (2015) Benefits of strict rest after acute concussion: A randomized controlled trial. Pediatrics 135: 1-11.

11. DiFazio M, Silverberg ND, Kirkwood MW, Bernier R, Iverson GL (2016) Prolonged activity restriction after concussion: Are we worsening outcomes? Clin Pediatr 55: 443-451.

12. Corwin DJ, Zonfrillo MR, Master CL, Arbogast KB, Grady MF, et al. (2014) Characteristics of prolonged concussion recovery in a pediatric subspecialty referral population. JPEDS 165: 1207-1215.

13. Taubman B, Mchugh J, Rosen F, Elci OU (2014) Repeat concussion and recovery time in a primary care pediatric office. J Child Neurol 14: 1607-1610.

14. Taubman B, Rosen F, McHugh J, Grady MF, Elci OU (2016) The Timing of Cognitive and Physical Rest and Recovery in Concussion. J Child Neurol 31: 1555-1560. [Crossref]

15. Ransom DM, Vaughan CG, Pratson L, Sady MD, McGill CA, et al. (2015) Academic effects of concussion in children and adolescents. Pediatrics 135: 1043-1050. [Crossref]

16. Taubman B (1987) Clinical experience. Is it always beneficial? Am J Dis Child 141: 674-675. [Crossref]

Copyright: (C2017 Taubman B. This is an open-access article distributed under the terms of the Creative Commons Attribution License, which permits unrestricted use, distribution, and reproduction in any medium, provided the original author and source are credited. 